\title{
Boolean and Smoothing of Discrete Polygonal Surfaces
}

\author{
Adam Updegrove $^{\mathrm{a}}$, Nathan M. Wilson ${ }^{\mathrm{b}}$, Shawn C. Shadden ${ }^{\mathrm{a}, *}$ \\ ${ }^{a}$ Department of Mechanical Engineering, University of California, Berkeley, CA \\ ${ }^{b}$ Open Source Medical Software Corporation, Santa Monica, CA
}

\begin{abstract}
The development of discrete solid models from imaging has become a common practice in reverse engineering. This has motivated the need for tools to combine and manipulate discrete boundary representation of objects. Despite the importance of this problem in computer aided engineering, there is a lack of robust and efficient open-source implementations of Boolean operations for discrete geometries. This paper discusses the development of new Visualization Tool Kit (VTK) classes for the Boolean and local mesh control of triangulated solid models. The implementation presented in this paper maintains the same base classes for Boolean operations in VTK version 6.2.0, but develops new procedures within these classes. Improvements are made on the robustness and performance of the Boolean algorithms for discrete surfaces. For example, for Boolean operations consisting of order 10,000 intersecting edges, the new implementation runs an order of magnitude faster than the current Boolean implementation in VTK, and is able to handle test cases the current implementation fails to handle. In addition, surface manipulation operations were created in order to deal with issues such as surface roughness, poor quality triangles, and sharp junctions that are often encountered in discrete solid modeling. These operations are implemented locally to give increased control. A unique smoothing method is also developed to address the issue of global model distortion common to prior smoothing procedures.
\end{abstract}

Keywords: Boolean, discrete solid model, smoothing, visualization toolkit

\section{Introduction}

Evolving technologies have shifted how computational solid models are often constructed. Increasingly, computer models are reverse engineered from imaging data, rather than being constructed from traditional CAD. For example, high resolution scanners provide a set of points, which can be connected to form a surface 5 representation of an object [1]. Using similar techniques, medical imaging (e.g., computed tomography, magnetic resonance imaging and ultrasound) can enable accurate construction of computer models representing internal organs, soft tissue, vasculature, and bones [2].

Motivation for the work herein stemmed from the need to create vascular models from medical image data, especially those suitable for computational modeling. Image-based vascular modeling is challenging given

\footnotetext{
${ }^{*}$ Corresponding author at: 5126 Etcheverry Hall, University of California, Berkeley, CA 94720-1740. Tel: +1510664 9800 Email address: shadden@berkeley.edu (Shawn C. Shadden)
} 
the natural tortuosity, branching and multi-scale nature of cardiovascular structures [3]. In order to develop a computer model from medical image data, two main methods are in use (1) direct 3D segmentation and (2) lofted 2D segmentation. In the former approach, a surface is evolved in 3D space to obtain an appropriate boundary representation (B-Rep) of the segmented region; in the later approach, the region being segmented is skeletonized by paths, and a B-Rep is formed from lofting together a series of $2 \mathrm{D}$ image segmentations along the skeletal paths [2]. Direct 3D segmentation typically results in discrete polygonal surfaces, whereas prior implementation of the lofted 2D segmentation method employed commercial software to create analytic (Non-Uniform Rational B-Spline) surfaces [4]. However, in order to develop and utilize open source tools, as well as to interface into a discrete solid modeling framework, we have recently developed a method to enable 2D image segmentations to be lofted and represented as a discrete polygonal surface [5], consistent with the output of most 3D segmentation softwares.

In many simulation-based modeling applications in engineering and design it is common to perform Boolean operations between objects or models. Such operations may be necessary in the image-based model construction process (e.g., combining members segmented separately), or in the development of a multidomain model from separate objects. One example is the virtual placement of a medical device into an anatomical model derived from image data.

There are a variety of Boolean implementations for B-Reps described in the literature. They can be classified in four categories by the computational approach: (1) tolerance and exact arithmetic, (2) approximate arithmetic, (3) volumetric, and (4) image space technqiues [6]. Tolerance and exact arithmetic methods both compute the intersection between two solids on their exact boundary, but contain different techniques for dealing with geometric robustness. Tolerances restrict floating point numbers to a specified decimal place for geometric tests; whereas, exact integer arithmetic methods convert floating point numbers to an integer-based system in which computations can be carried out exactly $[7,8,9,10]$. Approximate arithmetic methods reduce the computational complexity before running the geometric algorithm [11, 12]. This will make the computation simpler and quicker, but lacks the exactness of the previous approaches. results in a robust implementation; however, the boundary between the two solids is typically not resolved well in the output and a loss of geometric detail is seen [16]. Lastly, image space techniques take advantage of graphics hardware to quickly provide a boundary evaluation of the Boolean [17, 18]; these methods are typically used for object collision detection. Many of these algorithms use Layered Depth Images (LDIs) 40 as the volumetric methods in which the geometric detail is not exact; however, they do provide a fast and robust method to obtain a visual of the Boolean boundary [19, 20].

The Boolean implementations in literature also differ by the type of data used in the computation. Many implementations focus on NURBS surfaces [21, 6]; however, there are others that investigate the procedure 45

for polygonal surfaces [22], which have been the leading representation for discrete solid models derived 
from image data. There are a limited number of libraries providing open source, available, and usable Boolean operations for polygonal surfaces. The Visualization and Computer Graphics Library (VCG) has an implementation of the Boolean operation, which is implemented within the software MeshLab [23]. This is a volumetric implementation, and thus lacks the exactness of geometric detail described above. Another implementation is the GNU Triangulated Surface Library (GTS, http://gts.sourceforge.net), which we have found to be robust, but this package is no longer maintained and is difficult to include in software projects that require customization. VTK maintains an implementation for the Boolean of triangulated surfaces [24], however we have found that the implementation is not robust and often fails in various manners described below.

Rarely does the result of a Boolean give a surface that is ready for meshing and simulation, and typically other surface preparation methods must be performed. Besides defining faces for specification of boundary conditions or material properties, methods providing smoothing, blending, and manipulation of surfaces are necessary to give a solid that both accurately represents the image data and is valid for computational modeling. Moreover, discrete solid models obtained through image segmentation are often limited in quality by the resolution of the image data and inherent noise. Therefore surface manipulation tools are also necessary to improve the quality and representation of discrete solid models that serve the basis for quantitative postprocessing and simulation.

In this paper we describe the development of new VTK libraries for the Boolean, and local mesh control, of triangulated solid models. First the details of the developed Boolean procedures are discussed in Section 2. Each step of the Boolean is described followed by an analysis of the algorithm's performance. Next, customized surface manipulation operations created for local mesh control are presented in Section 3, and compared to current methods. Finally, concluding remarks are made in Section 4. The classes developed are included in the open-source SimVascular 2.0 software (simvascular.org); however, since they are implemented within the VTK framework they are modular and easily extendable elsewhere.

\section{Boolean Procedure}

VTK maintains an implementation for the Boolean of triangulated surfaces [24], however the current implementation is not robust and often fails in various manners as demonstrated in Fig. 1. Because of VTK's broad utility, modularity, and inclusion in numerous software packages, a decision was made to adapt on the VTK implementation to create a new, more robust Boolean procedure for model creation. New work of Mei and Tipper [25] and found in the GTS implementation.

The current VTK version 6.2.0 Boolean operation [24] consists of two separate VTK filters, vtkIntersectionPolyDataFilter for finding the intersection loops and re-triangulating the surfaces, and vtkBooleanOperationPolyDataFilter for determining the output surface. The implementation presented in this paper 
and functions implemented is located in the Appendix. For the new implementation, a computational approach following an exact boundary representation using a tolerance was chosen. The motivation for our work was the development of computational domains from medical image data in which the exactness of the model is paramount to correct results. Thus, an approximate or image space technique is not appropriate. A tolerance-based method was chosen because a number of VTK libraries currently use tolerances including vtkDelaunay2D, which is used within the Boolean.

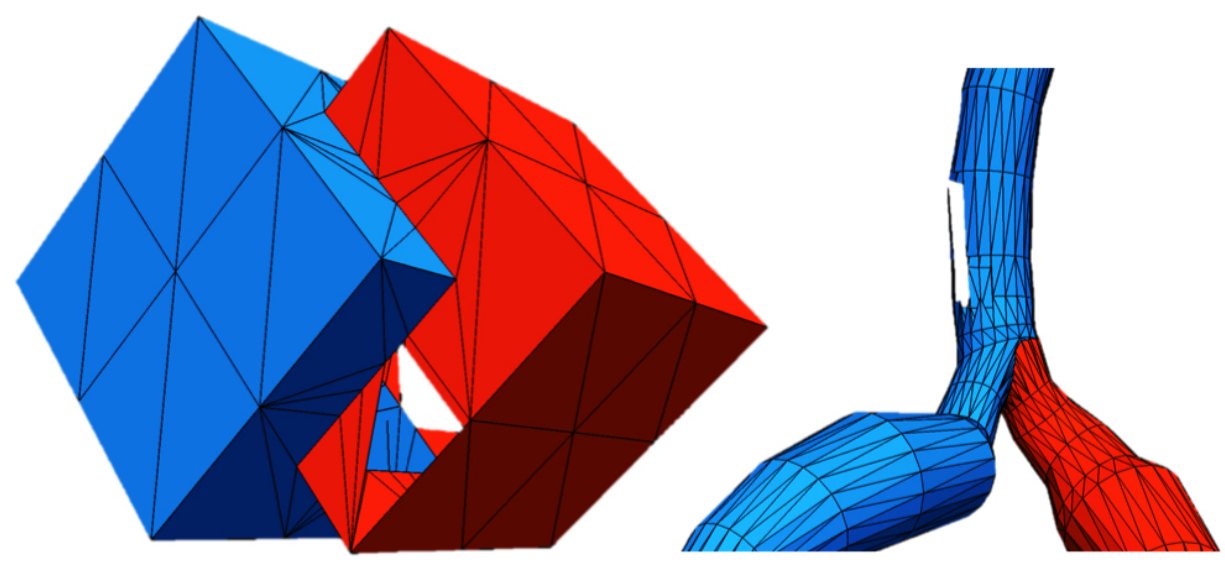

Figure 1: The current VTK implementation often fails due to incorrect intersection point surface origin determination (left) or due to incorrect sub-surface determination using a signed distance calculation (right). Issues such as these diminish the practical utility of this implementation.

\subsection{Overview}

A Boolean procedure takes as input two objects and outputs some combination of these objects. Henceforth, we denote the first input object as $A$ and the second as $B$. The possible Boolean operations between the objects are assumed to be two triangulated surface meshes. Moreover, these surfaces are considered B-Reps, or exterior surfaces, of volumetric objects in 3D space. Thus, one may view the Boolean as the union/intersection/difference of the enclosed volumes, subsequently restricted to the surface mesh. ${ }^{1}$

To explain the Boolean procedure, we require a couple of definitions. Intersection loops define where the intersection loops. The Boolean in general consists of (1) finding the intersection loops between the two objects, (2) separating the objects into appropriate sub-surfaces, and (3) determining the appropriate combination of the sub-surfaces for the desired Boolean. For a discrete polygonal Boolean, an additional step is required. After the intersection loops are found, the two input surfaces are re-triangulated to conform

\footnotetext{
${ }^{1}$ This interpretation holds for water-tight surfaces. As discussed further below, for open surfaces the Boolean is strictly between the surface objects.
} 
to the intersection loops. In this way, the respective sub-surfaces can be combined to give a valid output once the Boolean is performed. The following summarizes the computational steps in the Boolean process for discrete polygonal surfaces:

Intersection. Determine where the input surfaces intersect in space. This step creates the intersection loops that are used for re-triangulation and sub-surface determination (see Section 2.2).

Re-triangulation. Re-triangulate each surface near the intersection loops. The intersection loops are comprised of intersection points and lines on each surface, and each surface is re-triangulated separately (see Section 2.3).

Boolean. Determine the correct combination of sub-surfaces for output. The sub-surfaces are extracted based on their orientation relative to the intersection loops incident on the surfaces (see Section 2.4).

\subsection{Intersection}

The first step in the Boolean is finding intersection loops. The methods used to find intersection loops of two surfaces follows the work described in [24] with some modifications. Finding the intersection loops between two discrete surfaces is difficult because all individual intersecting cells between surfaces $A$ and $B$ must be found. Thus, intersections are found with the help of vtkOBBTree, an oriented bounding box tree class found in VTK. An oriented bounding box tree partitions space occupied by a discrete surface into subregions to enable better location queries. An oriented bounding box (OBB) is the smallest volume box that encloses a specified number of cells. The vtkOBBTree class contains a function ::IntersectWithOBBTree() that takes as input another vtkOBBTree to determine which OBBs from each surface intersect. For intersecting OBBs, the callback function ::FindTriangleIntersections() uses a function ::TriangleTriangeIntersection(), defined below, to find triangle intersections. An example of two oriented bounding box trees is displayed in Fig. 2.

The function ::TriangleTriangleIntersection() is an existing function in the VTK class vtkIntersectionPolyDataFilter, however this function does not provide information regarding intersection points or origin surfaces, which are necessary for most Boolean implementations. An intersection point is a point where two surfaces meet that is incident on the edge of a triangle from either surface. The origin surface is the input surface containing the triangle edge that the intersection point lies on (cf. Fig. 5). A new ::TriangleTriangleIntersection function was developed in order to track and maintain the intersection points and origin surfaces for later use, as described next.

The function ::TriangleTriangleIntersection() takes as input 2 sets of 3 points, with each set representing a triangle from each surface. A check is first performed to determine if all three points from one triangle

lie on one side of the supporting plane of the other triangle, excluding the possibility of intersection. The supporting plane is the plane containing the triangle of interest. If intersection is possible, the intersection line is found and restricted to the OBBs of the two triangles. The intersection line, 1 , is the line where the supporting planes of the two triangles intersect. Each edge, e, of each triangle from surface A is intersected 

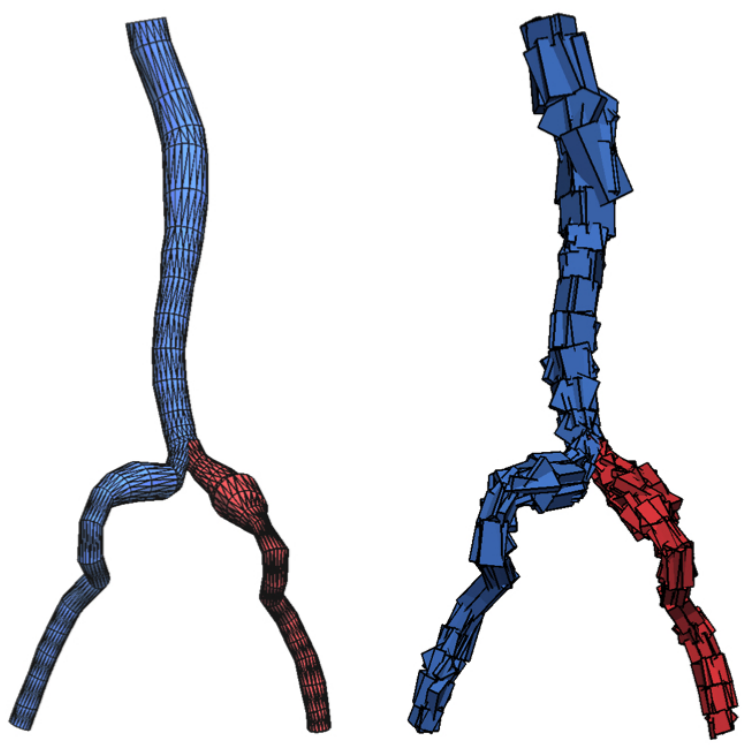

Figure 2: Left: The triangulated surface representation of a vascular model that was created by first developing a parent artery (blue) and branch artery (red) to be unioned. Right: oriented bounding boxes for each respective surface mesh. Each bounding box is specified to contain at most 10 surface triangles.

with $\mathbf{l}$ to give parametric values describing the intersection as shown in Fig. 3. Equations (1) and (2) give the intersection point, $P$, of the two line segments, and the parametric values $\left(\alpha_{1}, \alpha_{2}\right)$ describe where the intersection occurs on each line segment. The same procedure is followed with the triangles from surface B. The above procedure is only performed for triangles contained in intersecting OBBs.

$$
\begin{aligned}
& \underline{P}=\underline{a}+\alpha_{1}(\underline{b}-\underline{a}) \\
& \underline{P}=\underline{c}+\alpha_{2}(\underline{d}-\underline{c})
\end{aligned}
$$

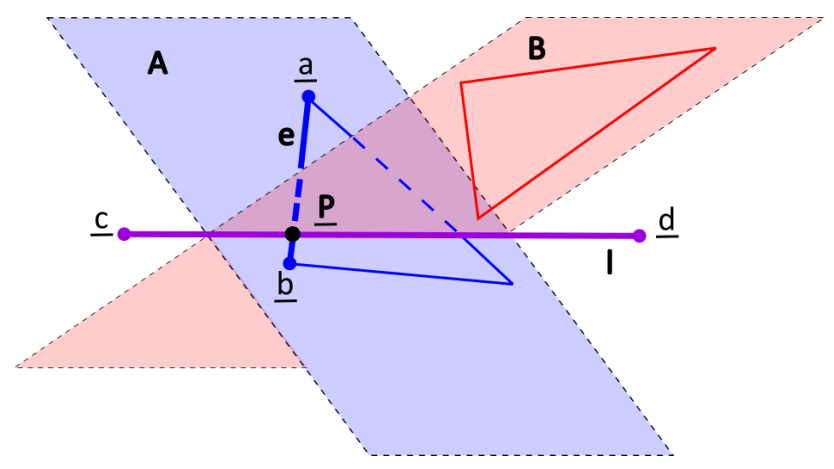

Figure 3: Line segment $\mathbf{e}$, which is an edge of the triangle from surface $\mathrm{A}$, has endpoints $a$ and $b$. This intersects with intersection line $\mathbf{l}$ with endpoints $c$ and $d$. The intersection gives two parametric values to determine where the intersection occurs along each respective line. Although the triangle from surface A intersects l, the triangles do not intersect because the triangle from surface B does not also intersect $\mathbf{l}$. 
A necessary, but not sufficient, condition for two triangles to intersect is that $\mathbf{l}$ must be intersected by at least 2 edges from each triangle. This can be determined by checking if the parametric values $\alpha_{1}$ and $\alpha_{2}$ are

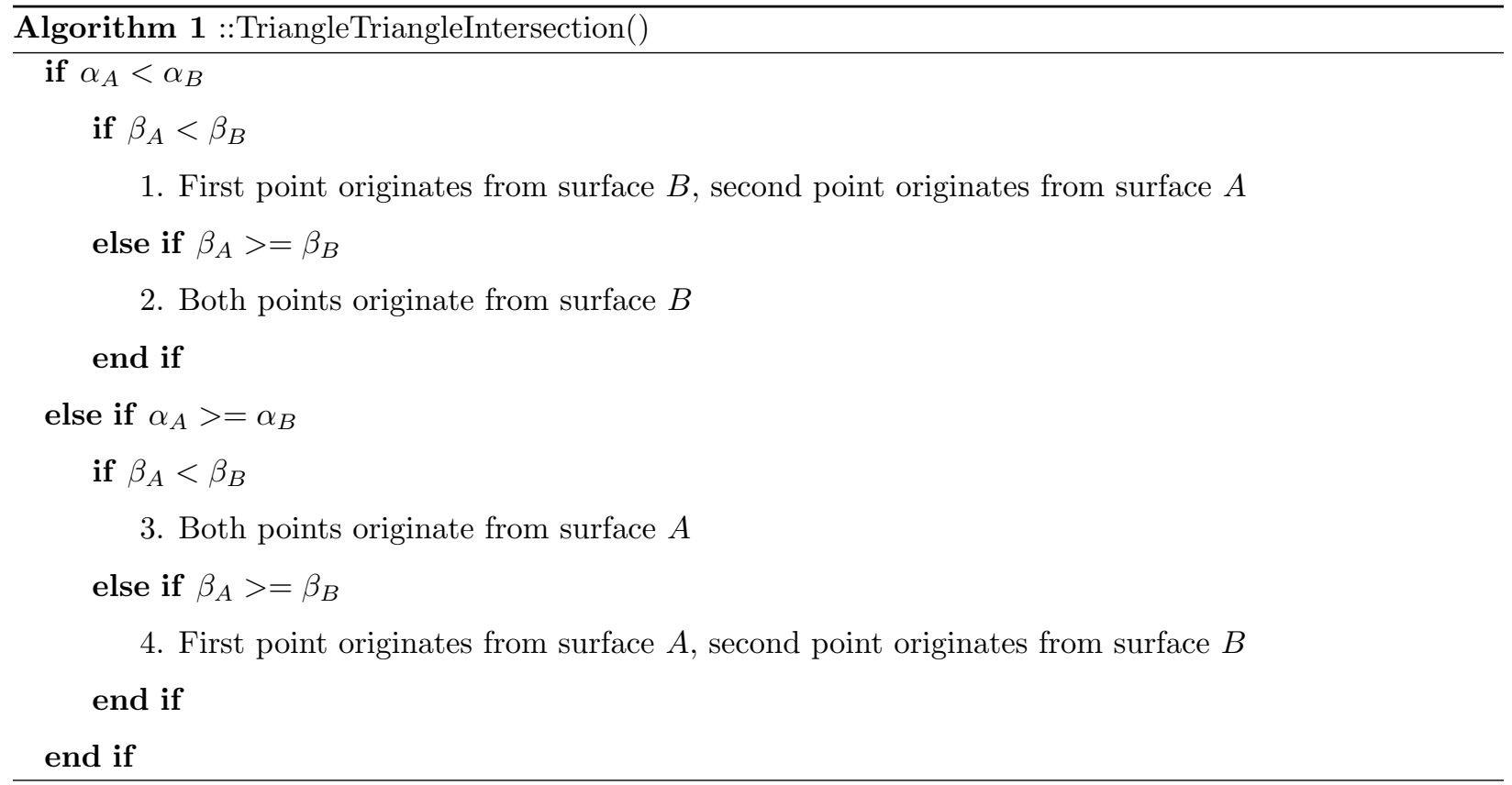

Figure 4: The different intersection types and how the parametric values determine the origin surface of the intersection points. The dashed line, $\mathbf{l}$, is the intersection line, and the arrow indicates the order in which the points are considered. The blue triangle is from surface $A$ and the red triangle is from surface $B$.

An intersection point may originate from: (1) surface $A,(2)$ surface $B$, or (3) both surfaces. Fig. 5 demonstrates the origin surface IDs given to intersection points from the intersection of two discrete spheres. These surface IDs determine whether a point is part of the boundary of an intersected triangle, which is needed in the re-triangulation step described below. The prior VTK implementation did not go through the process of finding the surface IDs. Instead, a distance calculation was used in the re-triangulation to determine whether a point is part of the triangle boundary. This calculation is an unnecessary step and 
often leads to an incorrect surface origin determination. Our approach provides a more robust solution to this problem. Each intersection point is stored in a vtkPoints object with its designated Origin Surface ID, and each intersection line is stored in a vtkCellArray. In addition, intersected triangles are marked for re-triangulation.

We note that for a Surface ID of 3, edges from each surface intersect. In this case, there are at least two triangle-triangle intersections that will both locate this intersection point. Therefore, we have added a check to eliminate duplicate intersection points. A point is flagged as duplicated if its distance from a prior intersection point is less than some specified tolerance (default $1 e-6$ ).

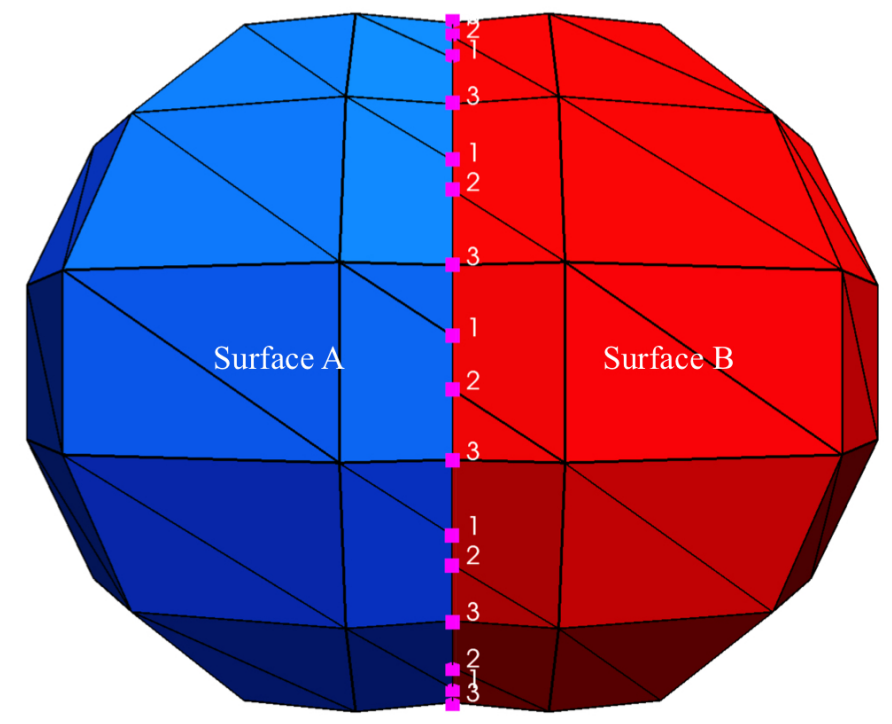

Figure 5: The intersection of two discrete spheres defined by intersection points with labeled origin surface IDs. 1 - intersection originates from Surface A; 2 - intersection originates from Surface B; 3 - intersection originates from both surfaces.

\subsection{Re-Triangulation}

The second step in the Boolean is the re-triangulation of both surfaces. The function ::SplitMesh() is the overarching re-triangulation function within the vtkIntersectionPolyDataFilter and the basic steps performed by this function are listed in Algorithm 2. This function is called separately for each input surface after the intersection loops have been found. It calls a function ::SplitCell(), which carries out the re-triangulation for intersected triangles. Algorithm 3 lists the general steps in the re-triangulation process. This new triangulation is found by first finding the cell loops of the split triangle. Fig. 6 and Algorithm 4 demonstrate the process of finding cell loops for an intersected triangle.

The intersected cells are re-triangulated according to Algorithm 3, and these are added to the retriangulated surface along with the existing non-intersected cells. The IDs of all cells adjacent to the intersection line are saved as vtkCellData for later use. At this point, the intersection is finalized, and the surfaces can be checked to ensure a proper triangulation was formed, i.e., that each edge adjoins an appro- 

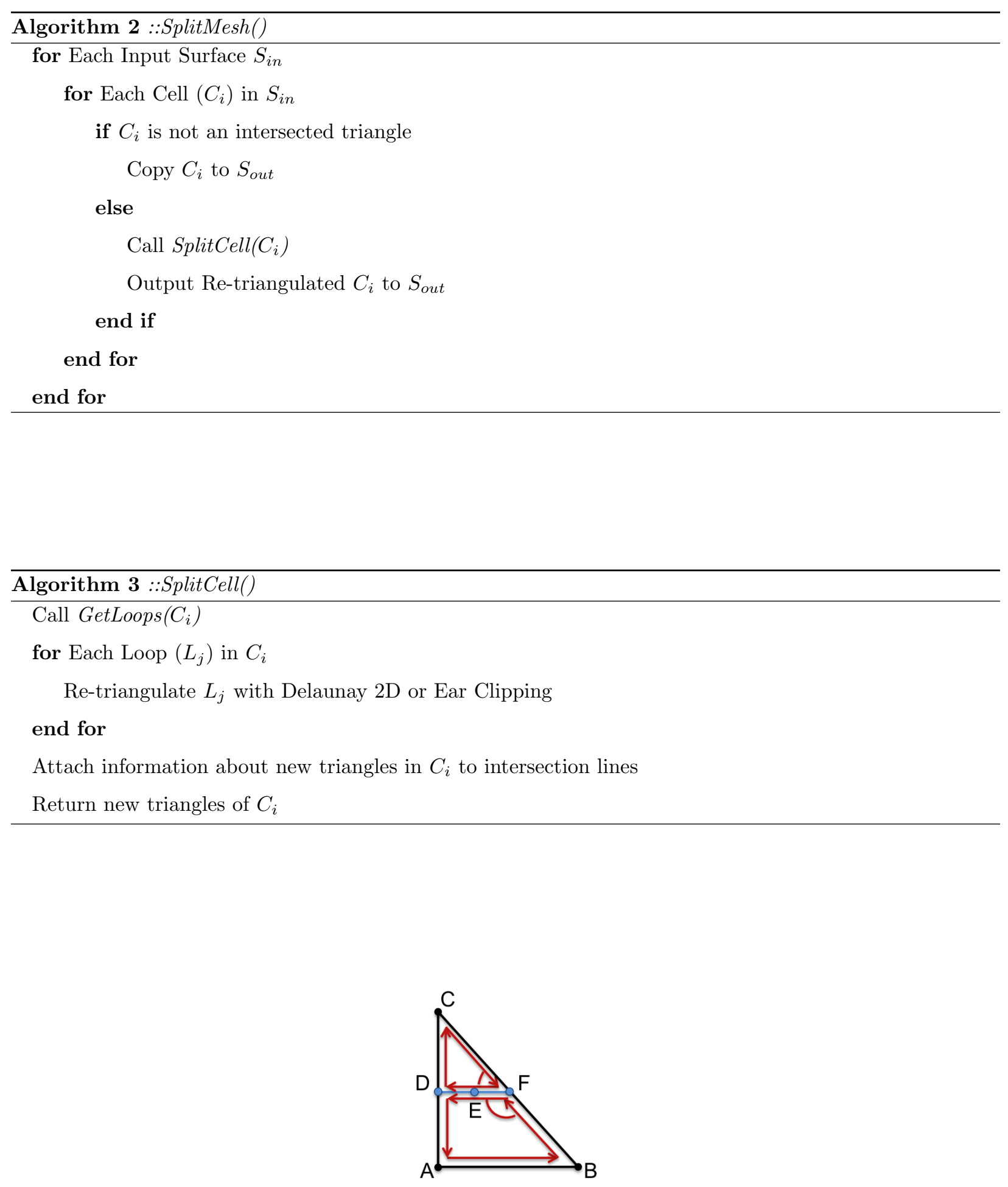

Figure 6: A triangle with an intersection splitting the cell into two loops. The loops are found using Algorithm 4. 


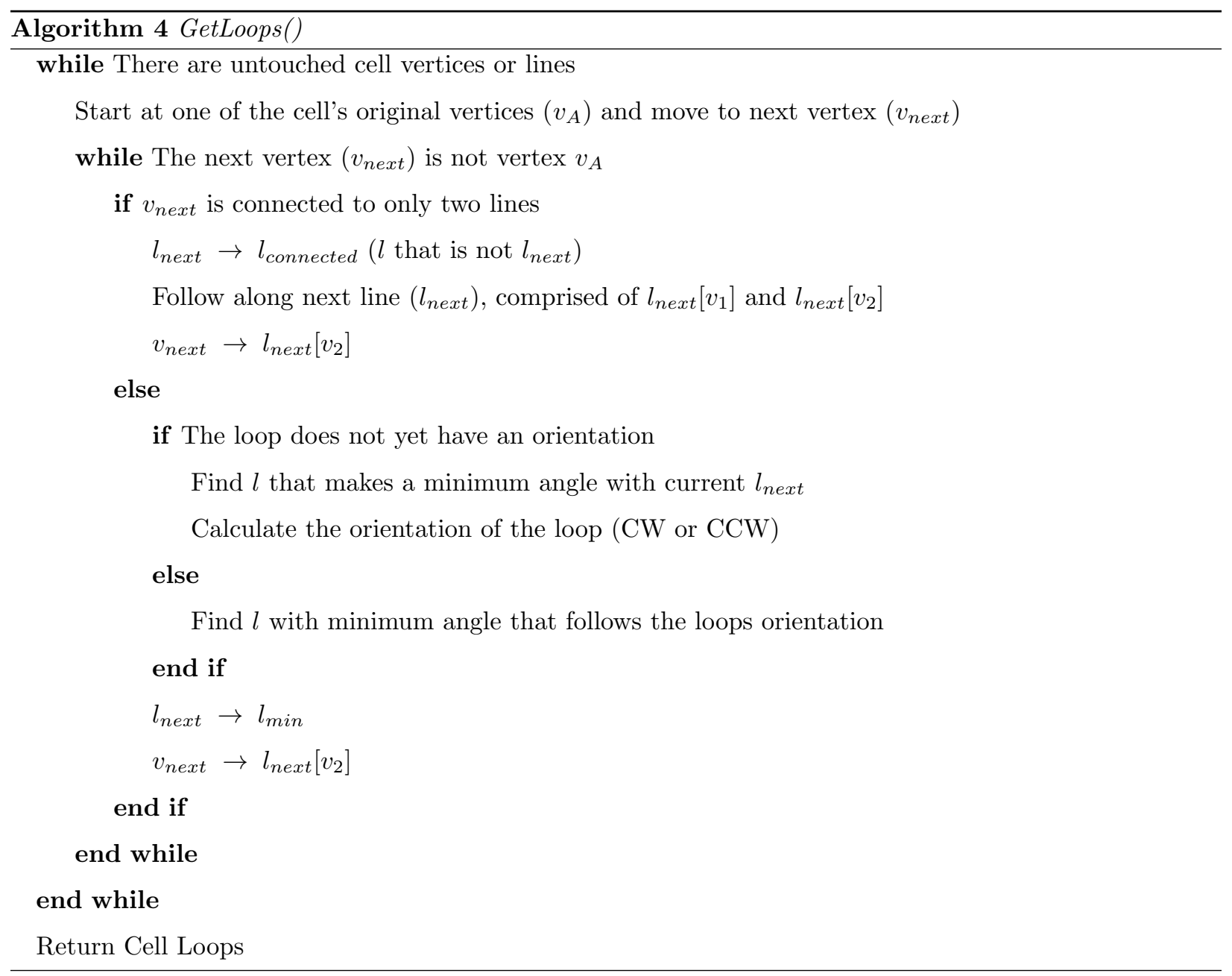




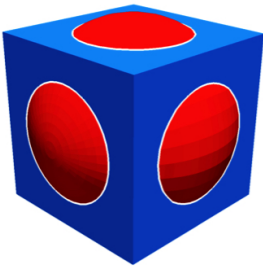

Hard Closed

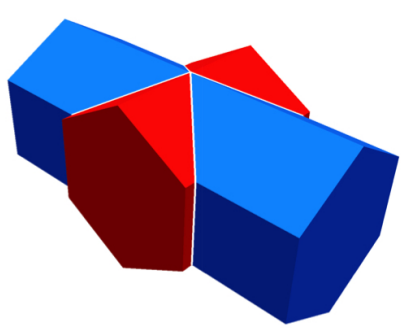

Soft Closed

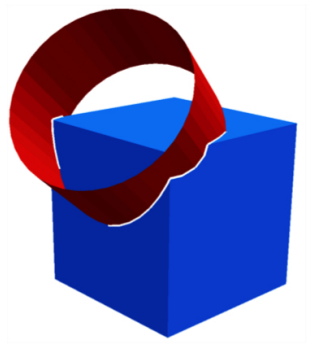

Open

Figure 7: The different intersection loop types possible. Left: An intersection containing 6 hard closed loops, Middle: An intersection containing 2 soft closed loops, Right: An intersection containing 1 open loop.

- Hard Closed Loop Intersection - Every intersection point is connected to two intersection lines, and the beginning point of each loop is the end point. There can be any number of intersection loops, but no intersection point can be connected to more or less than two intersection lines.

priate number of triangles. At the end of re-triangulation, the VTK class vtkIntersectionPolyDataFilter is completed.

\subsection{Boolean}

To obtain the correct Boolean output, a VTK class vtkBooleanOperationPolyDataFilter was developed. 175 The existing vtkBooleanOperationPolyDataFilter was found to have several deficiencies, and a new class was written in its place using alternative methods as described here. To begin the Boolean, the intersection loops are pre-processed to determine the type of intersection occurring between the surfaces. There are 3 intersection types for discrete polygonal surfaces [25], which are shown in Fig. 7. In what follows, we consider each intersection loop to be discretely represented by intersection points connected by intersection step above.

- Soft Closed Loop Intersection - Every intersection loop is closed; however, an intersection point can be connected to more than two lines. For example, in the intersection between two cylinders of the same radius, there are two soft closed intersection points that are connected to four intersection lines. The beginning point of each loop is still the end point of the loop, but points within the loop may be attached to more than, but not less than, two lines.

- Open Loop Intersection - Intersections do not form complete loops. There are points on the intersection lines that are only connected to one intersection line, and thus, are the ends of that loop. The beginning point is not the end point of the loop as the intersection loop is not closed.

It is possible to have a Boolean that gives rise to multiple intersection types. It is also worth noting that if the surfaces are water-tight it is not possible to have an Open Loop intersection. This is only possible 
for surfaces with free edges. We consider here a water-tight surface one in which every cell edge has two neighbor triangles. An open surface has at least one edge that has only one neighbor triangle; since portions of the surface are open, it may not be considered as enclosing a volume.

As pre-processing, the number of intersection loops and the type of intersection loops are found and stored. Then, each intersection loop is run through in an oriented manner to obtain the intersected subsurfaces partitioned by the intersection loops. The VTK class vtkBooleanOperationPolyDataFilter has a function ::GetBooleanRegions() that obtains the intersected sub-surfaces for each input, and is described in Algorithm 5.

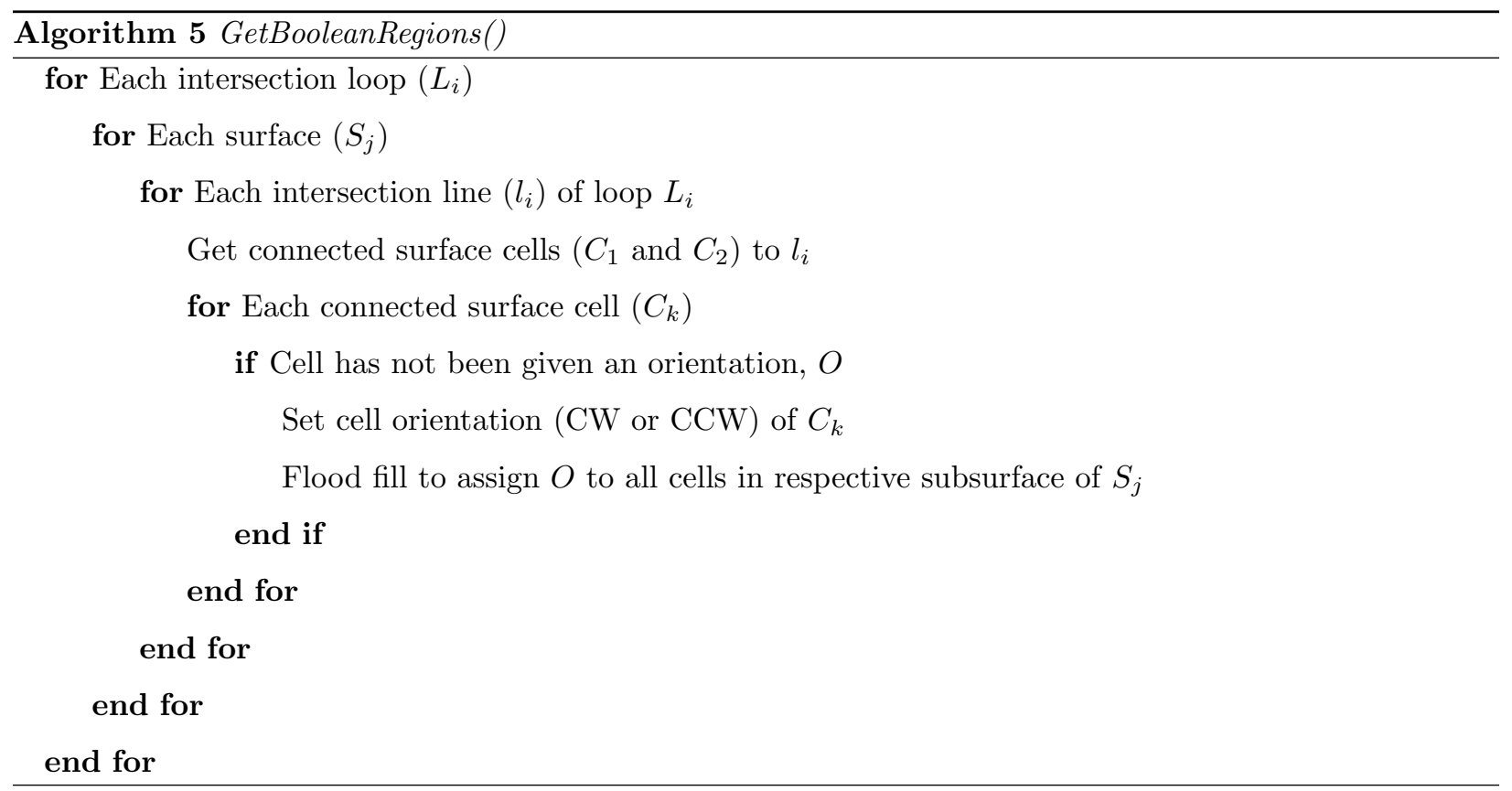

Figure 8: Orientation of cells adjacent to an intersection line. Cell to the left is oriented CCW and cell to the right is oriented $\mathrm{CW}$

The cells adjacent to the intersection loop lines are given an orientation based on their alignment when the intersection loop is transversed in a specified direction. For example, in Fig. 8, the intersection line is oriented bottom to top, making the cell to the left $\mathrm{CCW}$ and the cell to the right $\mathrm{CW}$. All cells attached to this first cell but not outside the containment of the intersection lines are assigned this orientation with the use of a flood fill algorithm that designates all connected elements inside the subsurface the same orientation. The boundaries for the flood fill are the intersection loops. The flood fill algorithm only runs 
once for every sub-surface-as initiated once the first element in that subsurface is considered. Fig. 9 displays the sub-surfaces defined by the intersection between a sphere and a cylinder. The desired Boolean (union, intersection, subtraction) is some combination of the sub-surfaces. The determination of which sub-surfaces to use is dependent on both the intersection loop types and the Boolean operation being performed. For the case when all intersection loops are hard closed loops, the determination is straightforward and follows the description in Fig. 9. To demonstrate a more practical application, Fig. 10 demonstrates the union of two arterial segments created from 2D segmentation [5] of medical image data.

\section{B}

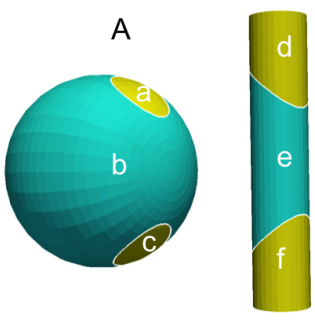

Sub-Surfaces

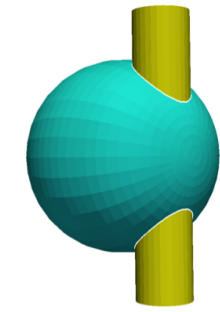

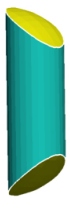

$A \cap B$

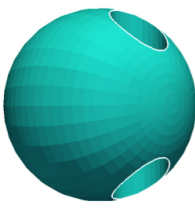

$A-B$
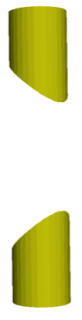

Figure 9: A sphere and cylinder intersection. The sphere and cylinder each have three sub-surfaces, $a, b, c$ and $d, e, f$. The sub-surfaces are colored by their Boolean orientation, yellow is CW and cyan is CCW. $A \cup B=b+d+f, A \cap B=a+c+e$, $A-B=b+e$, and $B-A=a+c+d+f$
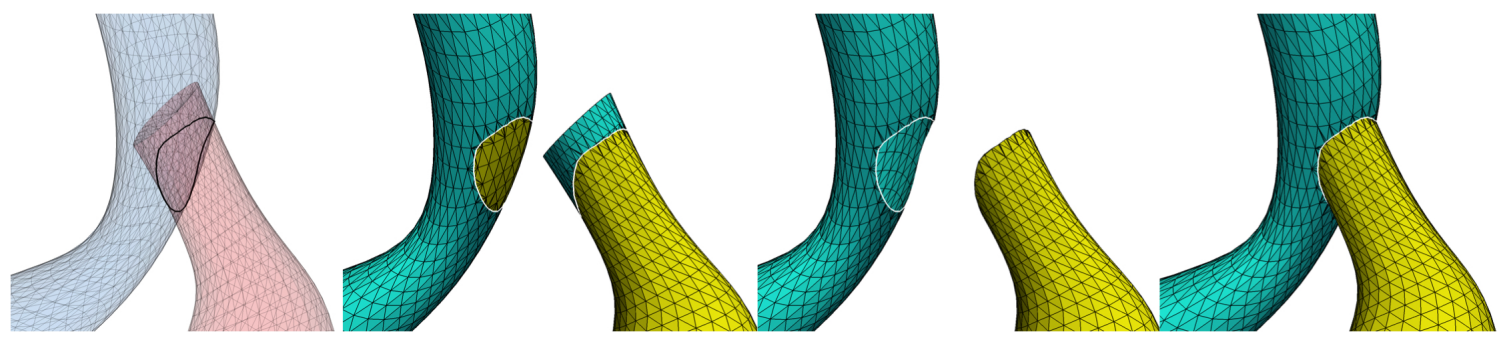

Figure 10: The union of two arteries give the combined vascular model. First, the intersection lines are computed. Each surface is re-triangulated and sub-surface orientations are determined. The sub-surfaces corresponding to a union operation are selected and unified to give the output polygonal surfaces.

\subsection{Special Cases: Open Loops and Soft Closed Loops}

The Boolean procedure is well defined for water-tight B-Reps since the inside and outside of these surfaces are known. For open surfaces (i.e., there are cell edges with no neighbors), the "inside" is less obvious. Therefore, for ease of understanding and to allow the possibility to perform a Boolean on open surfaces, the outside of an open surface is defined by the direction the normals are facing. The Boolean procedure implemented assumes that the direction of the normals is the exterior and the opposite direction is the interior. As shown in Fig. 7, the Boolean operation on open surfaces can lead to the case of an open intersection loop. This is one of the special cases considered in the Boolean procedure. The other special 
case arises when surfaces have similar sizes and they intersect at an identical surface point creating soft closed loops.

Open loop intersections.. In this scenario there are additional steps required to ensure the correct Boolean is output. The intersection and re-triangulation processes remain the same regardless of intersection type. At the beginning of the Boolean step, the loop intersection types (hard closed, soft closed, open) are characterized. In the case we have at least one open loop, the open loop(s) are sent to the back of the priority

queue for sub-surface definition. In this way, any closed or soft closed loops are processed first. The correct sub-surfaces are filled with correct orientations from the closed loops. Subsequently, open loops are processed and any remaining sub-surfaces are given an orientation in the same manner. Alternatively, in cases where there are only open intersection loops, the loops are processed sequentially; however, one surface will contain a sub-surface with no cells. This means two things: (1) the surface's other sub-surface contains all the cells, and (2) this is the sub-surface that needs to be used for the union. In this manner the union is specified to be composed of the larger portion of the sub-surfaces. Fig. 11 demonstrates the Boolean outputs in the case of open loop intersections. As shown, when open loop intersections occur, the Boolean is between the actual surfaces, and not the enclosed volume.

\section{Intersection Lines Union}

$A \cup B$
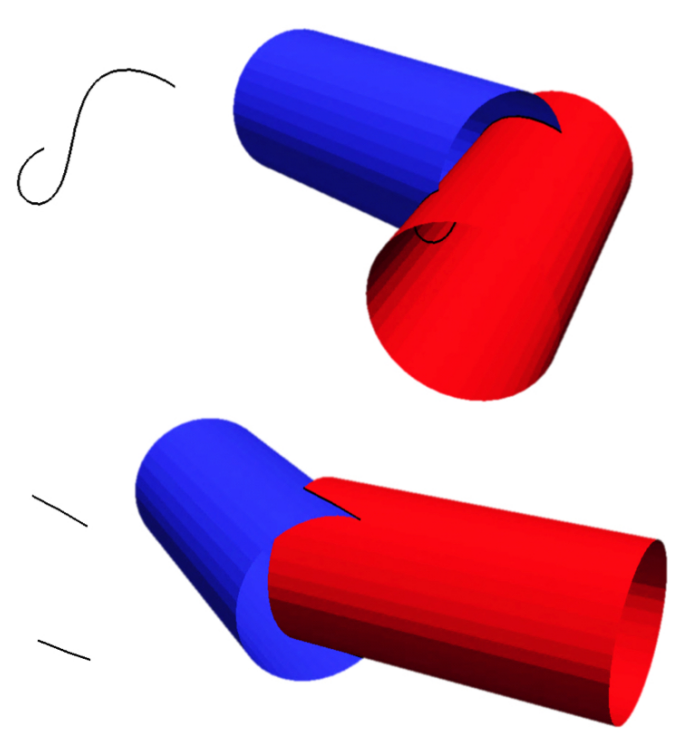

Intersection

$A \cap B$

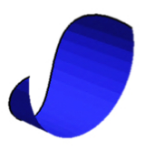

NULL

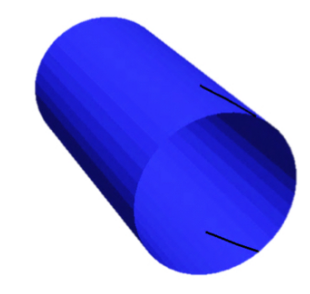

Difference

A - B

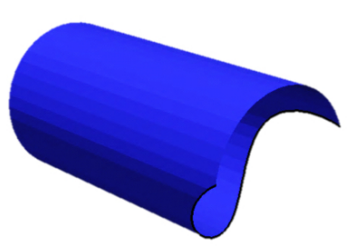

Figure 11: Booleans that result in open intersection loops. The top Boolean results in a union that has all of surface $B$ and an intersection comprised of only surface $A$. The bottom Boolean shows the case with two open intersection loops. The union retains all of both input surfaces and the intersection is a NULL surface.

Soft closed intersection loops.. This scenario is less common, but when it occurs there are special procedures

followed to ensure the correct Boolean output is achieved. Like the open loop case, the intersection and re- 
triangulation process are not affected. The specialized procedures come into play during the determination of intersection loop types. A soft closed intersection point (intersection point having more than two attached lines) indicates a soft closed loop. When this point is identified, there are multiple loops possible, and the correct intersection loops must be chosen. To do this, each of the attached lines are taken and the loops completed as potential loop candidates. Note that each loop may contain multiple soft closed intersection points and each possible route must be considered in forming potential loop candidates. Each possible loop is run through as is done during sub-surface determination (cf. Algorithm 5); however, there are two alterations. First, only cells of one orientation are used to fill regions (CCW in our implementation). Second, the number of regions filled is tracked. In the case that the loop candidate returns only one filled region, the correct loop has been found, and the remaining loop candidates do not need to be tested. After loop identification, the remaining steps are carried out in the same manner as hard closed loops. Fig. 12 depicts examples in which a Boolean results in soft closed loop intersections.

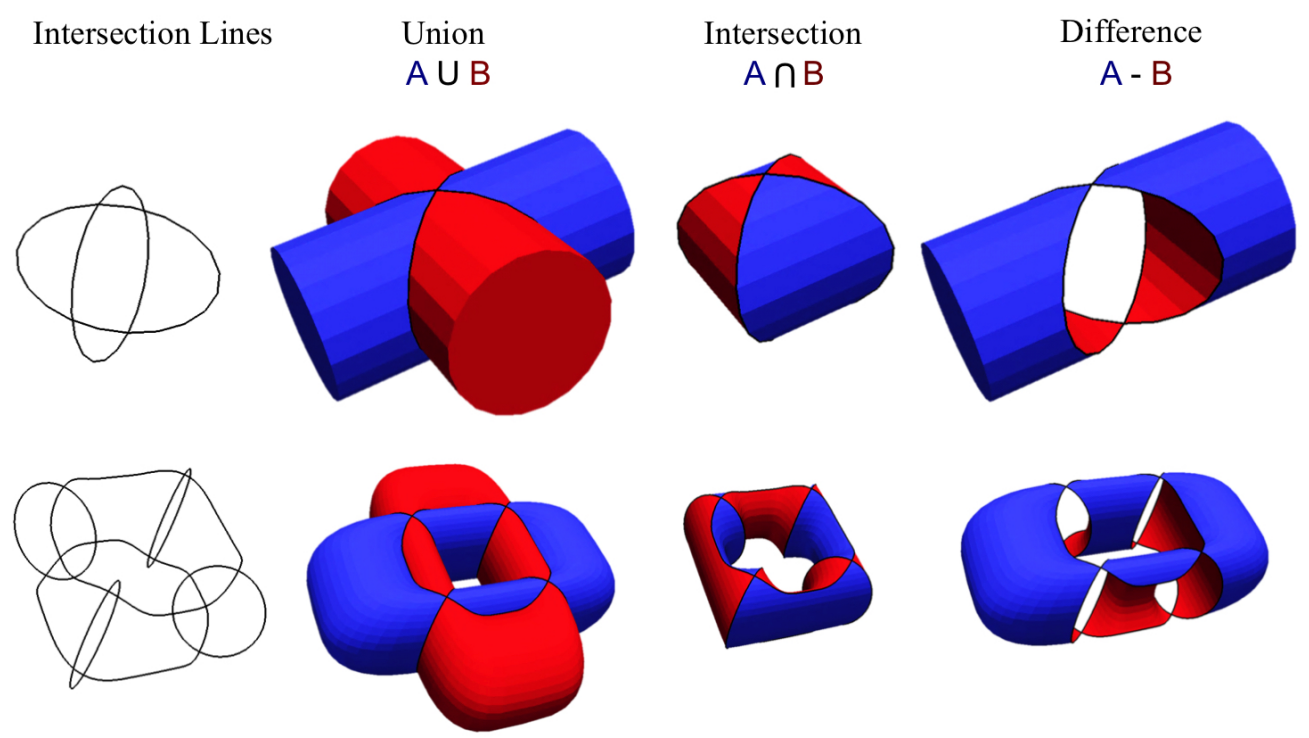

Figure 12: Booleans that result in soft closed intersection loops. The top intersection has two soft closed loops while the bottom intersection has four soft closed loops.

\subsection{Performance Improvements}

A brief analysis of the run time of Boolean procedures in 3D shows that the implementations described above perform in reasonable time. The worst case run time for a Boolean of polyhedral surfaces is $O\left(n \lambda^{2}+\right.$ $n \log (\Delta))$ time, where $n$ is the number of intersecting cells on surface $A$ and surface $B$. The density, $\lambda$, is defined as the smallest number such that the following holds true: Any ball $R$ intersects $\lambda$ edges $(e)$ belonging to the surface such that length $(e) \leq \operatorname{diam}(R) . \Delta$ is the spread of surface $A$ and $B$ and is defined as $\Delta=\frac{D}{d}$, where $D$ is the size of the smallest quadrant of the binary space partition (BSP) built on the cells. $d$ is the diameter of the smallest ball that intersects $k+2 N_{S D} \lambda+1$ edges of the surface. $k$ is the maximum number 

for Booleans when a large number of intersections are present.

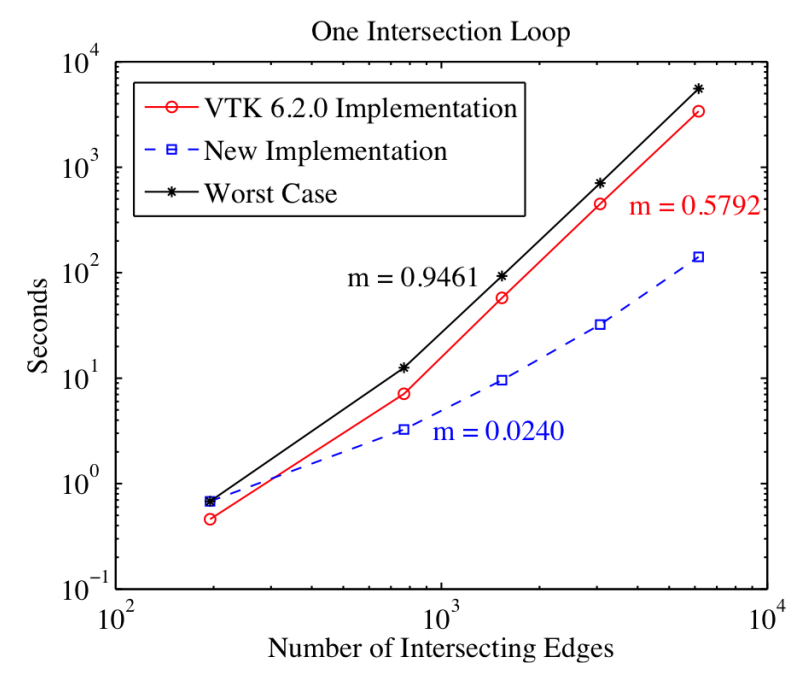

of cells for BSP tree quadrant and $N_{S D}$ is the number of spatial dimensions (in this case 3). See [26] for additional details on BSP trees and their use on polyhedral surfaces.

We present here two representative performance tests. For the first test (Fig. 13), there is only one intersection loop and the triangles on both input surfaces are of low aspect ratio (close to equilateral). In the second test (Fig. 13), there are two intersection loops and the triangles have high aspect ratios. To test the scaling of the algorithm, the cells for each surface were repeatedly subdivided as to increase the number of intersecting cells. In order to compute the worst case runtime (solid curve with $*$ ), the following assumptions were made. First, upon each subdivision, $\Delta$ is is assumed to increase by a factor of 2 . Second, upon each subdivision, for test 1 (Fig. 13), $\lambda$ was assumed to increase by a factor of two. For test 2 (Fig. 13), because of the high aspect triangles used, $\lambda$ was assumed to increase by a factor of 4 upon each subdivision. As shown in these figures, both prior and new implementations perform better than the assumed upper limit. However, the new implementation scales significantly better than the Boolean implementation in VTK version 6.2.0 [24] with an increasing number of intersecting edges. This performance improvement is realized for both test cases. In particular, for surfaces containing triangles of low aspect ratios, which is often the case in model construction, the new implementation runs close to two orders of magnitude faster

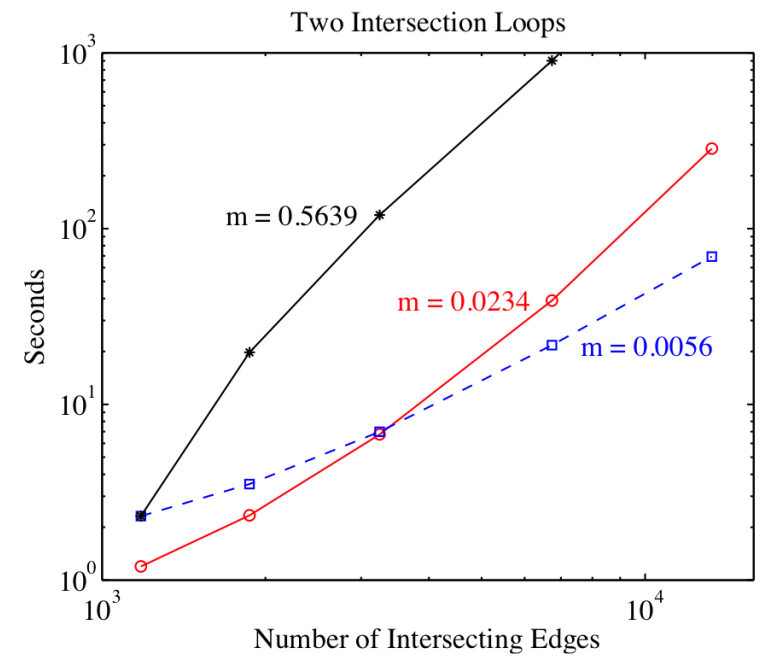

Figure 13: Performance plots for Boolean operations with one and two intersection loops. The developed implementation is compared to the VTK version 6.2.0 [24] implementation and the worst case performance for a discrete Boolean in 3D.

\section{Surface Manipulation Operations}

A surface output from a Boolean operation, such as the one described in Section 2, typically has sharp angles where the two input surfaces are united. Also in many practical applications, discrete B-Rep surfaces contain some amount of undesirable roughness since they are often generated from image data where analytic 
representation is not available. For either case, surface manipulation operations, which move points or alter point connectivity, are necessary to improve the final surface for its subsequent use.

In this work, three surface manipulation operations were developed: (1) smoothing, (2) decimation, and (3) subdivision. Smoothing attempts to lessen the angle difference between adjacent cells in the mesh. These values such as Taubin smoothing [28]. Decimation is the process of decreasing the number of triangles to represent the surface. Decimation is often desirable since it can decrease the number of poor quality elements and decrease the complexity of the model. While decreasing the number of facets, decimation also attempts to introduce the smallest amount of error possible. There are different ways in which to define error and simplify the surface [29]. The methods we developed use a quadric metric [30]. Subdivision works opposite to decimation, in that it introduces more cells into an existing surface. Like most surface operations, subdivision also has multiple implementation techniques [31]. The surface subdivision we developed is linear subdivision. For each edge of the mesh, a new node is added at its midpoint. Each triangle is divided into 4 new triangles, increasing the number of triangles by a factor of 4 .
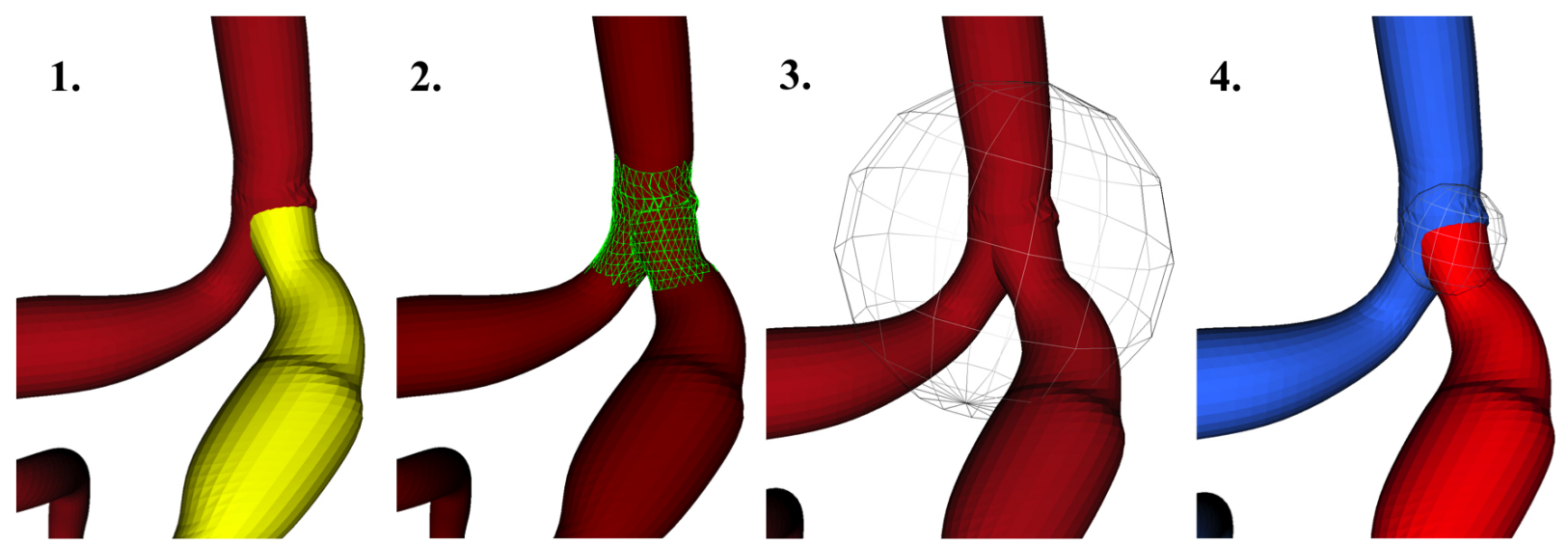

Figure 14: Four different cell selection types developed: (1) Any face or combination of faces can be selected. (2) Cells on the surface can be painted for selection. (3) A spherical region can be chosen. (4) The interface between two faces (red and blue) can be found and a sphere region with a specified radius can be selected around the interface.

Localized versions of the above operations were created because in most applications entire geometries do not need to be altered. Often only a specific, localized portion of a model needs smoothing. The localized methods were created for all mentioned surface manipulation operations by altering existing VTK filters to perform these operations. The exception to this was that an entirely new smoothing method was created, as described in Section 3.1. To be able to perform localized methods, node and cell selection procedures were created to identify portions of a model for manipulation (Fig. 14). The following four methods have been developed as part of a user interface to be able to interactively select regions: (1) A surface can be comprised of multiple faces and certain faces can be selected. (2) Cells can be painted on a surface which designate them as cells to be used. (3) A sphere with a specified radius and location can be placed and cells 
or nodes encapsulated within are selected. (4) With specialized algorithms, the interface between two faces can be found and then a sphere with a specified radius can be selected around this interface for selection. The above methods were customized to suit our application of developing discrete solid models for use with computational fluid dynamics (CFD).

\subsection{Constrained Smoothing}

Laplacian smoothing is often used on discrete geometries to decrease roughness, however smoothing can also degrade the accuracy of the representation. Of particular concern is that smoothing the B-Rep of an enclosed volume shrinks a geometry by nature. This occurs because a typical Laplacian smoothing assigns the new location of a point to be an average of the points sharing an edge with the specific point, which for one iteration is

$$
\underline{\mathbf{x}}_{\mathbf{i}}=\frac{1}{n} \sum_{j=0}^{n} \underline{\mathbf{x}}_{\mathbf{j}}
$$

where $n$ is the number of nodes sharing an edge with node $i$. Since all points are pulled toward their neighbors, as the number of smoothing iterations increases, each point will be pulled toward every other point in the mesh, and the surface will collapse inward. This is particularly a problem where the mesh object represents features of different scales. In such case, smoothing necessary to decrease roughness in locations of large scale features degrades the representation of small scale features needing to be preserved. For example, in vascular model construction, the shrinkage effect on large vessels may be insignificant over a few iterations, but the effect on small vessels may alter vessel area substantially, which can have dramatic consequences once the model is used for simulation.

To counteract this global shrinkage, a method was developed that solves for a surface that minimizes the error between the original mesh and the Laplacian smoothed mesh. Methods of this nature have recently been developed in work on computing watershed ridges [32]. The location of a smoothed point on the surface becomes the minimum of two equations in an optimization problem. Specifically, for each iteration, the coordinates of a new point $\underline{\mathbf{x}}_{\mathbf{i}}$ are described by the equations

$$
\begin{gathered}
\underline{\mathbf{x}}_{\mathbf{i}}-\left(\underline{\mathbf{x}}_{\text {original }}+\underline{\mathbf{w}}\right)=0 \text { where } \quad \underline{\mathbf{w}}=\left\|\underline{\mathbf{x}}_{\mathbf{i}}-\underline{\mathbf{x}}_{\text {original }}\right\| * w_{\text {user }} \\
\underline{\mathbf{x}}_{\mathbf{i}}-\frac{1}{n} \sum_{j=0}^{n} \underline{\mathbf{x}}_{\mathbf{j}}=0
\end{gathered}
$$

where $w_{\text {user }}$ is a user defined weighting between 0 and 1 used to penalize deviations from the original representation. If we apply these equations to all points, then we obtain the following matrix equations 


$$
\underbrace{\left[\begin{array}{cccccccc}
1 & 0 & 0 & \cdots & \cdots & \cdots & \cdots & 0 \\
0 & 1 & 0 & \ddots & & & & \vdots \\
0 & 0 & 1 & \ddots & \ddots & & & \vdots \\
\vdots & \ddots & \ddots & \ddots & \ddots & \ddots & & \vdots \\
\vdots & & \ddots & \ddots & \ddots & \ddots & \ddots & \vdots \\
\vdots & & & \ddots & \ddots & 1 & 0 & 0 \\
\vdots & & & & \ddots & 0 & 1 & 0 \\
0 & \cdots & \cdots & \cdots & \cdots & 0 & 0 & 1
\end{array}\right]}_{A_{1}}\left[\begin{array}{c}
x_{0}^{x} \\
x_{0}^{y} \\
x_{0}^{z} \\
\vdots \\
\vdots \\
x_{m}^{x} \\
x_{m}^{y} \\
x_{m}^{z}
\end{array}\right]=\underbrace{\left[\begin{array}{c}
x_{\text {original }}^{x}+w_{0} \\
x_{\text {original }}^{y}+w_{0} \\
x_{\text {original }}^{z}+w_{0} \\
\vdots \\
\vdots \\
x_{\text {original }}^{x}+w_{m} \\
x_{\text {original }}^{z}+w_{m} \\
x_{\text {original }}^{x}+w_{m}
\end{array}\right]}_{b_{1}}
$$

Since each point's coordinates has 3 components the total degrees of freedom of this system is $3 * m$, where $m$ is the number of points on the surface mesh. We note that the weighting is only applicable if the smoothing is done for more than one iteration. In many scenarios, it is desirable to have certain points that do not move at all. This can be implemented by setting the weighting for these points to 0 , or by leaving these points out of Equation (7). More generally, the weighting values can be varied among the points to locally constrain smoothing in a spatially varying manner.

To solve the above matrix equations (6) and (7), these matrices are concatenated vertically $A=\left[A_{1} ; A_{2}\right]$ and $b=\left[b_{1} ; b_{2}\right]$ and the extended system $A x=b$ is solved using the conjugate gradient method. Namely, the conjugate system $A^{T} A x=A^{T} b$ is solved, which corresponds to the minimization of $f(x)=\langle A x-b, A x-b\rangle$. That is, when the quadratic function $f(x)$ is minimum, the gradient is equal to zero

$$
\nabla_{x} f=2 A^{T}(A x-b)=0
$$

Fig. 15 shows the effect of the constrained smoothing on a select portion of a vascular model. When combined with localized decimation operations, the blending between the vessels increases. For a full blending of the branching vessel, these operations are also combined with decimation to give a smooth, natural transition 
between the vessels. Fig. 16 displays the effect of using constrained and non-constrained smoothing, demonstrating that constrained smoothing is able to create a smooth transition while maintaining otherwise high

\section{Conclusions}

A Boolean procedure for discrete polygonal surfaces was developed that greatly improves on algorithm runtime for discrete Booleans. This algorithm and implementation scales significantly better than the Boolean implementation in VTK version 6.2.0. The new methods were tested for cases with single and multiple intersection loops and surfaces with high and low aspect triangles. Additionally, specialized operations to locally improve surface quality were developed. This operations enable the ability to remove undesired roughness while not losing the essential features of a model. This was made possible by solving for a surface that minimizes the error between the original and smoothed mesh. This feature is important 

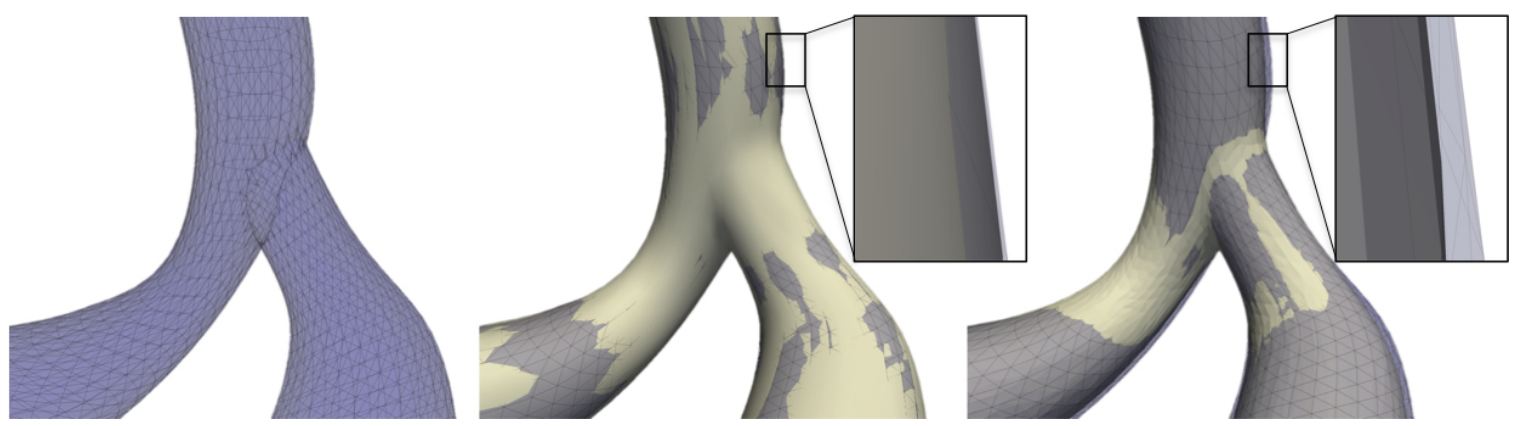

Figure 16: The original surface mesh (left) is smoothed using constrained smoothing. The constrained smoothed model (middle) is displayed along with the original mesh demonstrating the ability to create a smooth transition while retaining the original volume. Application of regular Laplacian smoothing (right) is not able to maintain the original volume.
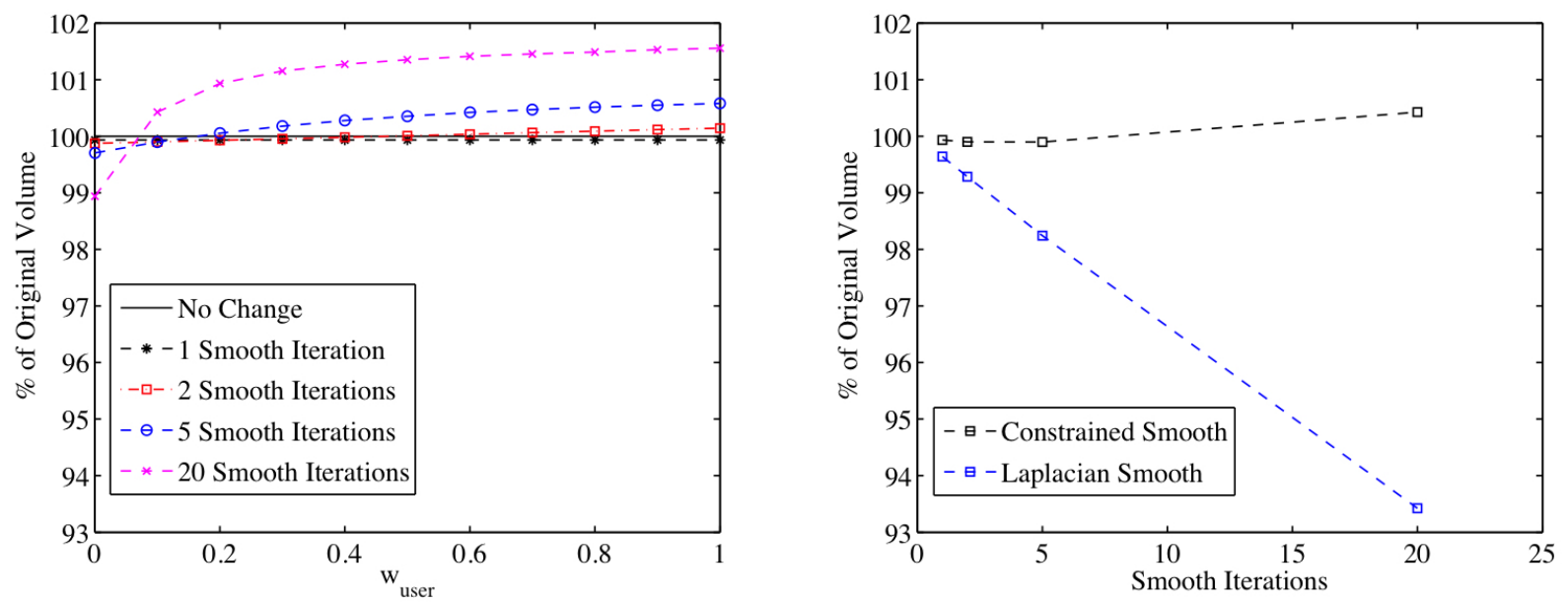

Figure 17: Left: The percentage of the original volume when constrained smoothing is performed for 1 , 2, 5 , and 20 iterations. Each case was attempted for user weightings from 0.1 to 1.0 . Right: Constrained smoothing using a weighting of 0.1 compared to the regular Laplacian smoothing.

in applications including computational fluid dynamics, where slight changes in the surface can significantly affect the results. Operations such as these are often essential when reverse engineering solid models from image data.

The Boolean in this paper was implemented using a tolerance, which was present throughout the VTK libraries. A possible improvement would be to implement an exact arithmetic method where the floating point numbers are first converted to integers to improve algorithmic robustness on geometric tests. All methods described above have been implemented as VTK classes, providing capability to plugin and be used with other VTK libraries. Besides making these methods more usable, this enables the surface operations to be used in coordination with other VTK filters. Currently, all of the operations described are available for use in the open source software project SimVascular 2.0; however, we plan to contribute related classes described herein to VTK for broader access and use within the community. 
[11] H. Biermann, D. Kristjansson, D. Zorin, Approximate boolean operations on free-form solids, in: Siggraph, Vol. 1, 2001, pp. 185-194.

\section{Acknowledgements}

This work was supported by the NSF (award number 1407834) and the NSF GRFP. We would like to thank Alison Marsden for her support with this project, and Mingcheng Chen for discussions on surface smoothing.

[1] G. Turk, M. Levoy, Zippered polygon meshes from range images, in: Proceedings of the 21st annual conference on Computer graphics and interactive techniques, ACM, 1994, pp. 311-318.

[2] N. Wilson, K. Wang, R. W. Dutton, C. Taylor, A software framework for creating patient specific geometric models from medical imaging data for simulation based medical planning of vascular surgery, in: Medical Image Computing and Computer-Assisted Intervention-MICCAI 2001, Springer, 2001, pp. $449-456$.

[3] L. Antiga, B. Ene-Iordache, A. Remuzzi, Computational geometry for patient-specific reconstruction and meshing of blood vessels from $\mathrm{mr}$ and ct angiography, Medical Imaging, IEEE Transactions on 22 (5) (2003) 674-684.

[4] I. Stroud, Boundary representation modelling techniques, Springer Science \& Business Media, 2006.

[5] A. Updegrove, N. M. Wilson, S. C. Shadden, Triangulated surface boolean operations for combining 2-d and 3-d image segmentation for patient-specific blood flow analysis, in: Summer Biomechanics, Bioengineering and Biotransport Conference, SB3C, 2015.

[6] A. Tayebi, J. Gomez Perez, F. Catedra, Boolean operations implementation over 3d parametric surfaces to be included in the geometrical module of an electromagnetic solver, in: Antennas and Propagation (EUCAP), Proceedings of the 5th European Conference on, IEEE, 2011, pp. 2137-2141.

[7] R. P. Banerjee, J. R. Rossignac, Topologically exact evaluation of polyhedra defined in csg with loose primitives, Computer Graphics Forum 15 (4) (1996) 205-217.

[8] S. Fang, B. Bruderlin, X. Zhu, Robustness in solid modelling: a tolerance-based intuitionistic approach, Computer-Aided Design 25 (9) (1993) 567-576.

[9] S. Fortune, C. J. Van Wyk, Efficient exact arithmetic for computational geometry, in: Proceedings of the ninth annual symposium on Computational geometry, ACM, 1993, pp. 163-172.

[10] S. Fortune, Polyhedral modelling with exact arithmetic, in: Proceedings of the third ACM symposium on Solid modeling and applications, ACM, 1995, pp. 225-234. 
[15] C. C. Wang, Approximate boolean operations on large polyhedral solids with partial mesh reconstruction, Visualization and Computer Graphics, IEEE Transactions on 17 (6) (2011) 836-849.

[16] L. P. Kobbelt, M. Botsch, U. Schwanecke, H.-P. Seidel, Feature sensitive surface extraction from volume data, in: Proceedings of the 28th annual conference on Computer graphics and interactive techniques,

[17] N. K. Govindaraju, M. C. Lin, D. Manocha, Fast and reliable collision culling using graphics hardware, Visualization and Computer Graphics, IEEE Transactions on 12 (2) (2006) 143-154.

[18] M. Teschner, S. Kimmerle, B. Heidelberger, G. Zachmann, L. Raghupathi, A. Fuhrmann, M.-P. Cani, F. Faure, N. Magnenat-Thalmann, W. Strasser, et al., Collision detection for deformable objects, Computer Graphics Forum 24 (1) (2005) 61-81.

[19] F. Faure, S. Barbier, J. Allard, F. Falipou, Image-based collision detection and response between arbitrary volume objects, in: Proceedings of the 2008 ACM SIGGRAPH/Eurographics Symposium on Computer Animation, Eurographics Association, 2008, pp. 155-162.

[20] M. Trapp, J. Döllner, Real-time volumetric tests using layered depth images, in: Proc. Eurographics,

[21] P. Yang, X. Qian, Direct boolean intersection between acquired and designed geometry, Computer-Aided Design 41 (2) (2009) 81-94.

[22] M. Schifko, B. JÃCEttler, B. Kornberger, Industrial application of exact boolean operations for meshes, in: Proceedings of the 26th Spring Conference on Computer Graphics, ACM, 2010, pp. 165-172.

[23] P. Cignoni, M. Callieri, M. Corsini, M. Dellepiane, F. Ganovelli, G. Ranzuglia, Meshlab: an open-source mesh processing tool., in: Eurographics Italian Chapter Conference, Vol. 2008, 2008, pp. 129-136.

[24] C. Quammen, C. Weigle, R. Taylor, M. II, Boolean Operations on Surfaces in VTK Without External Libraries, Insight Journal 3.00. 
[25] G. Mei, J. C. Tipper, Simple and robust boolean operations for triangulated surfaces, arXiv:1308.4434.

[29] P. S. Heckbert, M. Garland, Survey of polygonal surface simplification algorithms, Tech. rep., DTIC Document (1997).

[30] H. Hoppe, New quadric metric for simplifiying meshes with appearance attributes, in: Proceedings of the conference on Visualization'99: celebrating ten years, IEEE Computer Society Press, 1999, pp.

[31] K. Vlachkova, P. Terziev, A comparison of surface subdivision algorithms for polygonal meshes, in: Application of Mathematics in Technical and Natural Sciences, Vol. 1487, AIP Publishing, 2012, pp. 343-350.

[32] M. Chen, J. C. Hart, S. C. Shadden, Hierarchical watershed ridges for visualizing lagrangian coherent 


\section{Appendix}
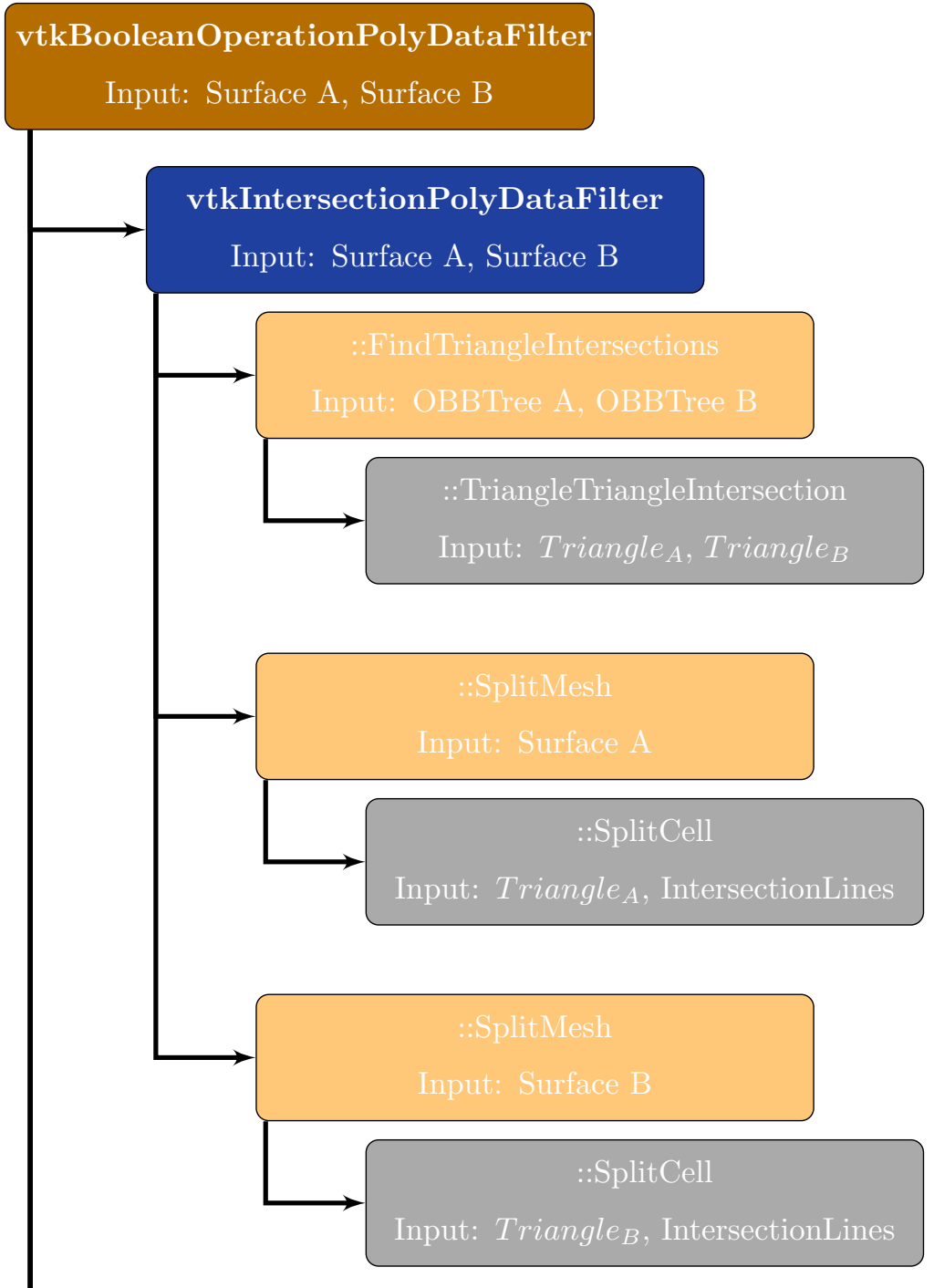

42

43

44

45

46

47

48

49

50

51

52

53

54

55

56

57

58

59

60

61

62

63 